\title{
UNIQUE FACTORIZATION OF COMPOSITIVE HEREDITARY GRAPH PROPERTIES
}

\author{
IZAK BROERE AND EWA DRGAS-BURCHARDT
}

\begin{abstract}
A graph property is any class of graphs that is closed under isomorphisms. A graph property $\mathcal{P}$ is hereditary if it is closed under taking subgraphs; it is compositive if for any graphs $G_{1}, G_{2} \in \mathcal{P}$ there exists a graph $G \in \mathcal{P}$ containing both $G_{1}$ and $G_{2}$ as subgraphs.

Let $H$ be any given graph on vertices $v_{1}, \ldots, v_{n}, n \geq 2$. A graph property $\mathcal{P}$ is $H$ factorizable over the class of graph properties $\mathbb{P}$ if there exist $\mathcal{P}_{1}, \ldots, \mathcal{P}_{n} \in \mathbb{P}$ such that $\mathcal{P}$ consists of all graphs whose vertex sets can be partitioned into $n$ parts, possibly empty, satisfying:

(1) for each $i$ the graph induced by the $i^{\text {th }}$ non-empty partition part is in $\mathcal{P}_{i}$, and

(2) for each $i$ and $j$ with $i \neq j$ there are no edges between the $i^{t h}$ and $j^{\text {th }}$ parts if $v_{i}$ and $v_{j}$ are non-adjacent vertices in $H$.

If a graph property $\mathcal{P}$ is $H$-factorizable over $\mathbb{P}$ and we know the graph properties $\mathcal{P}_{1}, \ldots, \mathcal{P}_{n}$, then we write $\mathcal{P}=H\left[\mathcal{P}_{1}, \ldots, \mathcal{P}_{n}\right]$. In such a case, the presentation $H\left[\mathcal{P}_{1}, \ldots, \mathcal{P}_{n}\right]$ is called a factorization of $\mathcal{P}$ over $\mathbb{P}$. This concept generalizes graph homomorphisms and $\left(\mathcal{P}_{1}, \ldots, \mathcal{P}_{n}\right)$-colorings.

In this paper we investigate all $H$-factorizations of a graph property $\mathcal{P}$ over the class of all hereditary compositive graph properties for finite graphs $H$. It is shown that in many cases there is exactly one such factorization.
\end{abstract}

Addresses:

Izak Broere ${ }^{(1)}$

Department of Mathematics

University of Johannesburg

P.O. Box 524

Auckland Park

2006 South Africa

Ewa Drgas-Burchardt ${ }^{(2)}$

Faculty of Mathematics, Computer Science and Econometrics

University of Zielona Góra

Prof. Z. Szafrana 4a, 65-516 Zielona Góra

Poland

Email addresses:

(1) izak.broere@up.ac.za. Present address: Department of Mathematics and Applied Mathematics, University of Pretoria, Pretoria, 0002 South Africa

(2) E.Drgas-Burchardt@wmie.uz.zgora.pl

Keywords: graph property, hereditary, compositive property, unique factorization, minimal forbidden graphs, reducibility

Subject Classification: 05C75, 05C15, 05C35

Abbreviated title: Unique factorization of graph properties 


\section{INTRODUCTION}

Factorizations of graphs and properties of graphs were first considered through investigations of colorings of graphs. Many researchers, in different branches of mathematics, have studied extensions of this topic such as $H$-coloring (to be homomorphic to a graph $H),\left(\mathcal{P}_{1}, \ldots, \mathcal{P}_{n}\right)$-coloring and joins in lattices of graph properties $[1,2,3,4]$. All these concepts are related to partitioning problems. The class of $n$-colorable graphs can be viewed as the class of those graphs $G$ for which there exists a partition of its vertex set into sets $V_{1}, \ldots, V_{n}$, so that each induced subgraph $G\left[V_{i}\right]$ is an edgeless graph. Allowing other possibilities than edgeless graphs, say having a graph $G\left[V_{i}\right]$ with property $\mathcal{P}_{i}$, we obtain the definition of the class of $\left(\mathcal{P}_{1}, \ldots, \mathcal{P}_{n}\right)$-colorable graphs. On the other hand, restricting requirements in the definition of $n$-colorability by requiring that edges between parts $V_{i}$ and $V_{j}$ are forbidden in $G$ for some listed pairs of indices from the set $\{1, \ldots, n\}$, we obtain the definition of $H$-colorability (to be homomorphic to the graph $H$ ). In this case $H$ is a graph with vertex set $\{1, \ldots, n\}$ satisfying that $i$ and $j$ are non-adjacent in $H$ if and only if edges between $V_{i}$ and $V_{j}$ are forbidden.

In this paper we put together these generalizations of $n$-colorings and we investigate the classes of graphs which have the special partitions which are described below.

A graph property $\mathcal{P}$ is any non-empty isomorphic closed subclass of the class of all finite non-isomorphic graphs having at least one vertex $\mathcal{I}$. The set $\mathcal{I}$ is called a trivial graph property. A graph property $\mathcal{P}$ is hereditary if it is closed under taking subgraphs, $\mathcal{P}$ is additive if for any two graphs $G_{1}, G_{2} \in \mathcal{P}$ the (disjoint) union of $G_{1}$ and $G_{2}$ is also in $\mathcal{P}$ and $\mathcal{P}$ is compositive if for any two graphs $G_{1}, G_{2} \in \mathcal{P}$ there exists a graph in $\mathcal{P}$ containing both $G_{1}$ and $G_{2}$ as subgraphs. Note that every additive graph property is compositive. We denote the class of all hereditary graph properties and all hereditary and compositive graph properties by $\mathbf{L}$ and $\mathbf{L}^{c}$ respectively. For instance, the graph properties $\mathcal{S}_{k}, \mathcal{O}^{k}$, where $\mathcal{S}_{k}=\{G \in \mathcal{I}: \Delta(G) \leq k\}$ and $\mathcal{O}^{k}=\{G \in \mathcal{I}: G$ is $k$-colorable $\}$ are hereditary and additive (and thus compositive) for each $k \in \mathbb{N}$. On the other hand, a graph property that contains $K_{3}$, all forests and all graphs obtained by taking the disjoint union of $K_{3}$ and a forest is hereditary and compositive but not additive. Moreover, $\mathcal{O}_{c}^{k}=\{G \in \mathcal{I}: G$ is a connected $k$-colorable graph $\}$ is an example of a graph property which is compositive but not additive and not hereditary.

Let $n \geq 2$ and let $H$ be a graph with vertex set $\left\{v_{1}, \ldots, v_{n}\right\}$. Let $\mathcal{P}_{1}, \ldots, \mathcal{P}_{n}$ be graph properties, that is, classes of graphs closed under isomorphisms. We define a graph property $\mathcal{P}$ to be $H$-factorizable over a class of graph properties $\mathbb{P}$, if there exist properties $\mathcal{P}_{1}, \ldots, \mathcal{P}_{n} \in \mathbb{P}$ such that $\mathcal{P}$ consists of all the graphs whose vertex sets can be partitioned into $n$ parts, possibly empty, such that:

(1) for each $i$ the graph induced by the $i^{t h}$ non-empty partition part is in $\mathcal{P}_{i}$, and

(2) for each $i$ and $j$ with $i \neq j$ there are no edges between the $i^{t h}$ and $j^{\text {th }}$ parts if $v_{i}, v_{j}$ are non-adjacent vertices in $H$.

If $\mathcal{P}$ is $H$-factorizable with factors $\mathcal{P}_{1}, \ldots, \mathcal{P}_{n}$, then we write $\mathcal{P}=H\left[\mathcal{P}_{1}, \ldots, \mathcal{P}_{n}\right]$. In view of this definition it is easy to see that the classes of $n$-colorable, $\left(\mathcal{P}_{1}, \ldots, \mathcal{P}_{n}\right)$-colorable and $H$-colorable graphs are $K_{n}[\mathcal{O}, \ldots, \mathcal{O}], K_{n}\left[\mathcal{P}_{1}, \ldots, \mathcal{P}_{n}\right]$ and $H[\mathcal{O}, \ldots, \mathcal{O}]$ respectively where $\mathcal{O}$ is the graph property to be edgeless and $K_{n}$ is the complete graph on $n$ vertices. Moreover, $\overline{K_{n}}\left[\mathcal{P}_{1}, \ldots, \mathcal{P}_{n}\right]$, where $\overline{K_{n}}$ denotes the complement of $K_{n}$, is the join of properties $\mathcal{P}_{1}, \ldots, \mathcal{P}_{n}$ in the lattice of hereditary graph properties closed under taking disjoint unions [4]. 
$H$-factorizability was first considered for the class of additive hereditary graph properties $[5,6]$. For given $H$-factorizable graph property $\mathcal{P}$, the uniqueness of its $H$-factorization, and the cardinality of minimal forbidden graph family were investigated in these papers. Such problems were earlier intensively studied for $n$-colorability, $\left(\mathcal{P}_{1}, \ldots, \mathcal{P}_{n}\right)$-colorability and $H$-colorability $[7,8,9,10,11,12,13]$.

In this paper we explore the $H$-factorizability of the class of compositive hereditary graph properties. We prove the uniqueness of $H$-factorizations in this class based on a unique representation of a graph in terms of the family of prime graphs. This representation can be constructed in accordance with Gallai's result [14] which we discuss in Section 2. Section 2 also includes some definitions and observations.

In Section 3 we introduce the basic concept of factorizability. Besides special features of the prime graph $\overline{K_{2}}$, some useful remarks are also formulated.

Section 4 investigates the uniqueness of an $H$-factorization for a prime graph $H$.

Section 5 contains the main results of the paper. It is readable immediately after Section 3 and familiarization with the definition of a dominating vertex which is given before Lemma 6 .

\section{Preliminaries}

All graphs considered in this paper are finite, undirected and simple. Let $G$ denote a graph with the vertex set $V(G)$ and the edge set $E(G)$. For a given $v \in V(G)$ let $N_{G}(v)$ and $\operatorname{deg}_{G}(v)$ denote the open neighborhood of $v$ and the degree of $v$ in the graph $G$ respectively. The maximum degree and the minimum degree in $G$ taken over all vertices of $G$ will be denoted by $\Delta(G)$ and $\delta(G)$. We write $\bar{G}, K_{n}$ and $C_{n}$ for the complement of $G$, a complete graph and a cycle of order $n$ respectively. For given graphs $G_{1}, \ldots, G_{n}$ and a graph $H$ with $V(H)=\left\{v_{1}, \ldots, v_{n}\right\}$, we will use the symbol $H\left[G_{1}, \ldots, G_{n}\right]$ to denote the graph whose vertex set is the union of $V\left(G_{1}\right), \ldots, V\left(G_{n}\right)$ and whose edge set consists of the union of $E\left(G_{1}\right), \ldots, E\left(G_{n}\right)$ with the additional edge set $\left\{\{x, y\}: x \in V\left(G_{i}\right), y \in\right.$ $\left.V\left(G_{j}\right),\left\{v_{i}, v_{j}\right\} \in E(H)\right\}$. To emphasize the special role of $H$ in this definition, we call it the base graph of $H\left[G_{1}, \ldots, G_{n}\right]$. Note that $K_{n}\left[G_{1}, \ldots, G_{n}\right]$ is the disjoint union of the graphs $G_{1}, \ldots, G_{n}$, frequently denoted by $G_{1} \cup \cdots \cup G_{n}$. Moreover, we adopt the notation $\overline{K_{n}}[G, \ldots, G]=n G$

A graph $G$ is homomorphic to a graph $H$ if there exists a mapping $\varphi: V(G) \rightarrow V(H)$ such that $\{u, v\} \in E(G)$ implies $\{\varphi(u), \varphi(v)\} \in E(H)$. Such a mapping is said to be a homomorphism from $G$ to $H$. A bijection $\varphi$ from $V(G)$ onto $V(H)$ in which $\{u, v\} \in E(G)$ if and only if $\{\varphi(u), \varphi(v)\} \in E(H)$ is called an isomorphism from $G$ to $H$ and in such a case we say that $G$ is isomorphic to $H$ and we write $G=H$. An isomorphism from $G$ to $G$ is called an automorphism (of $G$ ). We shall use the notation $G_{1} \subseteq G_{2}$ to denote the fact that $G_{1}$ is isomorphic to a subgraph of $G_{2}$; in such a case we often say that $G_{1}$ is a subgraph of $G_{2}$.

Let $[n]=\{1, \ldots, n\}$. If $V(H)=\left\{v_{1}, \ldots, v_{n}\right\}$ and $V(G)=\left\{x_{1}, \ldots, x_{m}\right\}$ and $\varphi$ is a homomorphism (or isomorphism with $n=m$ ) from $H$ to $G$, then we often simplify the notation by writing $\varphi:[n] \rightarrow[m]$ instead of $\varphi: V(H) \rightarrow V(G)$ and $\varphi(i)=j$ instead of $\varphi\left(v_{i}\right)=x_{j}$.

Following [14], a set $W$ of vertices of a graph $H$ is a module in $H$ if for any two vertices $x, y \in W$, the equality $N_{H}(x) \backslash W=N_{H}(y) \backslash W$ is satisfied. The trivial modules in $G$ are $V(G), \emptyset$ and the singletons. A graph having only trivial modules is called prime. We denote by PRIME the subclass of $\mathcal{I}$ of prime graphs on at least two vertices. It is 
worth mentioning that PRIME contains exactly one complete graph $K_{2}$ and exactly one disconnected graph $\overline{K_{2}}$. Moreover, there is no prime graph on three vertices.

We now present the main tool, a result of Gallai which describes the modular decomposition of an arbitrary graph, which is to be used in this paper.

Theorem 1. [14] Let $G$ be any graph with at least two vertices. Then exactly one of the following conditions holds:

(1) $G$ is disconnected and can be uniquely decomposed into its connected components,

(2) $\bar{G}$ is disconnected and can be uniquely decomposed into complements of connected components of $\bar{G}$,

(3) $G$ and $\bar{G}$ are connected and there is some $U \subseteq V(G)$ and a unique partition $\Pi$ of $V(G)$ such that

(a) $|U| \geq 4$,

(b) the subgraph of $G$ induced by $U$ is a maximal prime induced subgraph of $G$, and

(c) every part $S$ of the partition $\Pi$ is a module in $G$ with $|S \cap U|=1$.

There does not seem to be a convention on how to describe the modular decomposition of a graph given in Theorem 1. Thus we give the most convenient way for the presentation of our results.

Remark 1. Each graph $G \in \mathcal{I}$ on at least two vertices can be uniquely constructed by taking $|V(G)|$ copies of $K_{1}$, using the operation $H\left[G_{1}, \ldots, G_{n}\right], n \geq 2$ with $H$ a prime base graph and with $G_{1}, \ldots, G_{n}$ graphs obtained in previous steps. The uniqueness is understood to be up to automorphisms of the base graph and features of the special base graphs $K_{2}$ and $\overline{K_{2}}$.

We now describe how to apply Theorem 1 to build the prime graph $H$ mentioned in Remark 1 for a given graph $G$. If $G(\bar{G})$ is disconnected with components $G_{1}, \ldots, G_{s}, s \geq 2$, then we use the prime graph $H=\overline{K_{2}}\left(H=K_{2}\right.$ respectively) $s-1$ times. To be more precise, $G=\overline{K_{2}}\left[G_{i_{1}}, \overline{K_{2}}\left[G_{i_{2}}, \overline{K_{2}}\left[\ldots, \overline{K_{2}}\left[G_{i_{s-1}}, G_{i_{s}}\right] \ldots\right]\right]\right]\left(G=K_{2}\left[\overline{G_{i_{1}}}, K_{2}\left[\overline{G_{i_{2}}}, K_{2}\left[\ldots, K_{2}\left[\overline{G_{i_{s-1}}}\right.\right.\right.\right.\right.$, $\left.\left.\left.\overline{G_{i_{s}}}\right] \ldots.\right]\right]$ respectively) where $i_{1}, i_{2}, \ldots, i_{s}$ is an arbitrary permutation of the numbers from $[s]$. The structure of $G$ does not depend on the permutation used but on properties of $\overline{K_{2}}$ $\left(K_{2}\right)$ used as base graphs in an application of the definition. If none of the above cases hold (i.e. if $G$ and $\bar{G}$ are connected), then we find maximal modules in $G$ different from $V(G)$, say $V_{1}, V_{2}, \ldots, V_{p}$. It is known that $V_{1}, V_{2}, \ldots, V_{p}$ are pairwise disjoint sets and they form a partition of $V(G)$. The last base graph $H$ used in the construction has $\left\{V_{1}, \ldots, V_{p}\right\}$ as vertex set with two vertices $V_{r}$ and $V_{s}$ adjacent in $H$ if and only if at least one vertex from $V_{r}$ is adjacent in $G$ to at least one vertex from $V_{s}, r, s \in[p]$. It is evident that such $H$ is prime with at least four vertices and $V(H)$ can play the role of the set $U$ in condition (3) of Theorem 1. At the same time $V_{1}, \ldots V_{p}$ is the unique partition $\Pi$ in Theorem 1. Repeating the procedure for graphs induced in $G$ by $V_{1}, \ldots, V_{p}$ or for components of $G$ (complements of components of $\bar{G}$ ) in the above cases, we obtain the next prime base graphs of the construction. The procedure terminates if all graphs $G\left[V_{i}\right], i \in[p]$ or all components of $G$ (complements of components of $\bar{G}$ ) are isomorphic to $K_{1}$.

Readers familiar with the notion of the modular decomposition tree of a graph $[14,15]$ will see that the described construction corresponds to this tree. We focus on the structure between modules and describe it from the top in the language of a known product of graphs. Remark 1 implies the following observation. 
Remark 2. Let $G, G_{1}, \ldots, G_{n}, G_{1}^{\prime}, G_{2}^{\prime}, \ldots G_{m}^{\prime} \in \mathcal{I}$ and $H_{1}, H_{2}$ be prime graphs. If $G=$ $H_{1}\left[G_{1}, \ldots, G_{n}\right]=H_{2}\left[G_{1}^{\prime}, \ldots, G_{m}^{\prime}\right]$ then $H_{1}$ is isomorphic to $H_{2}$. Moreover, if $H_{1}$ has at least three vertices, then there exists an automorphism $\varphi$ of $H_{1}$ such that for each $i \in[n]$, the graph $G_{i}$ is isomorphic to the graph $G_{\varphi(i)}^{\prime}$.

$\mathcal{G}$ is a generating set for a hereditary graph property $\mathcal{P}$ if $\mathcal{G} \subseteq \mathcal{P}$ and every graph in $\mathcal{P}$ is a subgraph of some $G \in \mathcal{G}$. A generating set is ordered if its elements can be listed as $G_{1}, G_{2}, \ldots$ such that $G_{i}$ is a subgraph of $G_{i+1}$ for each permissible $i$. For example, for each $G \in \mathcal{I}$, the set $\{G\}$ is a generating set for the hereditary and compositive graph property consisting of all subgraphs of $G$.

A special case of a generating set for $\mathcal{P} \in \mathbf{L}$, the set of $\mathcal{P}$-maximal graphs denoted $M(\mathcal{P})$, is $\{G \in \mathcal{I}: G \in \mathcal{P}$ and $G+e \notin \mathcal{P}$ for each $e \in E(\bar{G})\}$. The following theorem joining the notions introduced above was verified in [10].

Theorem 2. [10] For any graph property $\mathcal{P}$, the following are equivalent:

(1) $\mathcal{P} \in \mathbf{L}^{c}$

(2) $\mathcal{P}$ has a generating set; moreover, for any graph $L \in \mathcal{P}$ and any generating set $\mathcal{G}$ for $\mathcal{P}$, the set $\{G \in \mathcal{G}: L \subseteq G\}$, denoted by $\mathcal{G}[L]$, is also a generating set,

(3) $\mathcal{P}$ has a (finite or infinite) ordered generating set,

(4) $\mathcal{P}$ has a (finite or infinite) ordered generating set $\left\{H_{1}, H_{2}, \ldots\right\}$ in which $H_{i}$ is $\mathcal{P}$-maximal and it is an induced subgraph of $H_{i+1}$ for all permissible $i$.

Let $\mathcal{P}_{1}, \ldots, \mathcal{P}_{n} \in \mathbf{L}^{c}, G \in \mathcal{I}$ and let $H$ be a graph with $V(H)=\left\{v_{1}, v_{2}, \ldots, v_{n}\right\}$. An $H\left[\mathcal{P}_{1}, \ldots, \mathcal{P}_{n}\right]$-partition of $G$ is defined as a partition $\left(V_{1}, \ldots, V_{n}\right)$ of $V(G)$ such that the existence of $\left\{x_{i}, x_{j}\right\} \in E(G)$ with $x_{i} \in V_{i}, x_{j} \in V_{j}, i \neq j$ implies the existence of $\left\{v_{i}, v_{j}\right\} \in E(H)$ and $G\left[V_{i}\right] \in \mathcal{P}_{i}$ or $V_{i}=\emptyset$ for $i \in[n]$. The symbol $H\left[\mathcal{P}_{1}, \ldots, \mathcal{P}_{n}\right]$ denotes the class of all graphs possessing an $H\left[\mathcal{P}_{1}, \ldots, \mathcal{P}_{n}\right]$-partition. In other words, $H\left[\mathcal{P}_{1}, \ldots, \mathcal{P}_{n}\right]$ consists of all graphs $H\left[G_{1}, \ldots, G_{n}\right]$ such that $G_{i} \in \mathcal{P}_{i}, i \in[n]$, and all their subgraphs. Evidently the condition $\mathcal{P}_{1}, \ldots, \mathcal{P}_{n} \in \mathbf{L}^{c}$ forces that $H\left[\mathcal{P}_{1}, \ldots, \mathcal{P}_{n}\right] \in \mathbf{L}^{c}$. For $\mathcal{P}=H\left[\mathcal{P}_{1}, \ldots, \mathcal{P}_{n}\right]$ we say that $H\left[\mathcal{P}_{1}, \ldots, \mathcal{P}_{n}\right]$ is an $H$-factorization (or, briefly, a factorization) of $\mathcal{P}$ over $\mathbf{L}^{c}$ and $\mathcal{P}_{1}, \mathcal{P}_{2}, \ldots, \mathcal{P}_{n}$ are $H$-factors (or, briefly, factors) of this factorization.

Let $\mathcal{P}_{1}, \ldots, \mathcal{P}_{n} \in \mathbf{L}^{c}$, and let $H$ be a graph with $V(H)=\left\{v_{1}, \ldots, v_{n}\right\}$. Next let $\mathcal{P}=H\left[\mathcal{P}_{1}, \ldots, \mathcal{P}_{n}\right]$ and suppose there exists a $\mathcal{P}$-maximal graph $G$ such that each $H\left[\mathcal{P}_{1}, \ldots, \mathcal{P}_{n}\right]$-partition $\left(V_{1}, \ldots, V_{n}\right)$ of $G$ satisfies $V_{i} \neq \emptyset$ for all $i \in[n]$. Then $H\left[\mathcal{P}_{1}, \ldots, \mathcal{P}_{n}\right]$ is called a proper $H$-factorization of $\mathcal{P}$ over $\mathbf{L}^{c}$ and the set of all $\mathcal{P}$-maximal graphs having the desired property is denoted by $M^{*}\left(H\left[\mathcal{P}_{1}, \ldots, \mathcal{P}_{n}\right]\right)$.

For example, let $\mathcal{P}=P_{3}\left[\mathcal{Q}, \mathcal{Q}^{\prime}, \mathcal{Q}\right]$, where $\left\{K_{2}\right\}$ and $\left\{K_{1}\right\}$ are generating sets for $\mathcal{Q}$ and $\mathcal{Q}^{\prime}$ respectively. Then $P_{3}\left[K_{2}, K_{1}, K_{2}\right] \in M^{*}\left(P_{3}\left[\mathcal{Q}, \mathcal{Q}^{\prime}, \mathcal{Q}\right]\right)$, which shows that this $P_{3}$-factorization of $\mathcal{P}$ is indeed proper.

We now formulate an obvious remark which appeared in [12] for the case $H=K_{n}$.

Remark 3. Let $\mathcal{P}_{1}, \ldots, \mathcal{P}_{n} \in \mathbf{L}^{c}$ and let $H$ be a fixed graph. Every graph $G \in M^{*}\left(H\left[\mathcal{P}_{1}, \ldots\right.\right.$, $\left.\left.\mathcal{P}_{n}\right]\right)$ has the form $H\left[G_{1}, \ldots, G_{n}\right]$ with $G_{i}$ a $\mathcal{P}_{i}$-maximal graph for all $i \in[n]$.

Note that for each graph $G \in M^{*}\left(H\left[\mathcal{P}_{1}, \ldots, \mathcal{P}_{n}\right]\right)$ and any supergraph $G^{\prime}$ of $G$ in $\mathcal{I}$, we have in each $H\left[\mathcal{P}_{1}, \ldots, \mathcal{P}_{n}\right]$-partition $\left(V_{1}, \ldots, V_{n}\right)$ of $G^{\prime}$ that $V_{i} \neq \emptyset$ for all $i \in[n]$. It follows that if $H\left[\mathcal{P}_{1}, \ldots, \mathcal{P}_{n}\right]$ is a proper $H$-factorization of $\mathcal{P}$, then $M^{*}\left(H\left[\mathcal{P}_{1}, \ldots, \mathcal{P}_{n}\right]\right)$ is a generating set for $\mathcal{P}$. 
In the light of Theorem 1 we can see that, if $H$ is a graph on at least two vertices, then each $H$-factorization of a given graph property $\mathcal{P}$ can be viewed as a composition of $G$ factorizations with $G$ being prime graphs. To get a feeling for arguing with an extendend structure, we start with the following lemma.

Lemma 1. Let $H_{1}$ and $H_{2}$ be prime graphs with $V\left(H_{1}\right)=\left\{v_{1}, \ldots, v_{n}\right\}, V\left(H_{2}\right)=\left\{v_{1}^{\prime}, \ldots v_{m}^{\prime}\right\}$, and let $H_{1}\left[\mathcal{P}_{1}, \ldots, \mathcal{P}_{n}\right], H_{2}\left[\mathcal{P}_{1}^{\prime}, \ldots, \mathcal{P}_{m}^{\prime}\right]$ be proper factorizations of $\mathcal{P}$ over $\mathbf{L}^{c}$. Then $H_{1}=H_{2}$ and consequently $n=m$. Moreover, if $n \geq 3$ and $G \in M^{*}\left(H_{1}\left[\mathcal{P}_{1}, \ldots, \mathcal{P}_{n}\right]\right) \cap$ $M^{*}\left(H_{2}\left[\mathcal{P}_{1}^{\prime}, \ldots, \mathcal{P}_{n}^{\prime}\right]\right)$, and $\left(V_{1}, \ldots, V_{n}\right)$ is an arbitrary $H_{1}\left[\mathcal{P}_{1}, \ldots, \mathcal{P}_{n}\right]$-partition of $G$, and $\left(V_{1}^{\prime}, \ldots V_{n}^{\prime}\right)$ is an arbitrary $H_{2}\left[\mathcal{P}_{1}^{\prime}, \ldots, \mathcal{P}_{n}^{\prime}\right]$-partition of $G$, then there exists an automorphism $\varphi:[n] \longrightarrow[n]$ of $H_{1}$ such that $V_{i}^{\prime}=V_{\varphi(i)}, i \in[n]$.

Proof. By points (4) and (2) of Theorem 2 we can construct a generating set for $\mathcal{P}$ consisting of only $\mathcal{P}$-maximal graphs from the set $M^{*}\left(H_{1}\left[\mathcal{P}_{1}, \ldots, \mathcal{P}_{n}\right]\right) \cap M^{*}\left(H_{2}\left[\mathcal{P}_{1}^{\prime}, \ldots, \mathcal{P}_{n}^{\prime}\right]\right)$. It follows from Remark 3 that each such graph $G$ satisfies $G=H_{1}\left[G_{1}, \ldots, G_{n}\right]=H_{2}\left[G_{1}^{\prime}, \ldots, G_{n}^{\prime}\right]$ and using Remark 2, by the assumption that $H_{1}, H_{2}$ are prime, we know that $H_{1}$ is isomorphic to $H_{2}$.

Let $n \geq 3$ and $\left(V_{1}, \ldots, V_{n}\right),\left(V_{1}^{\prime}, \ldots, V_{n}^{\prime}\right)$ be the assumed partitions of $G \in M^{*}\left(H_{1}\left[\mathcal{P}_{1}, \ldots, \mathcal{P}_{n}\right]\right)$ $\cap M^{*}\left(\bar{H}_{2}\left[\mathcal{P}_{1}^{\prime}, \ldots, \mathcal{P}_{n}^{\prime}\right]\right)$. It follows that $G=H_{1}\left[G\left[V_{1}\right], \ldots, G\left[V_{n}\right]\right]=H_{1}\left[G\left[V_{1}^{\prime}\right], \ldots, G\left[V_{n}^{\prime}\right]\right]$. The assertion now follows from Remark 2 .

\section{3. $\mathcal{C}$-REDUCIBILITy}

For a given graph $H$ with $V(H)=\left\{v_{1}, \ldots, v_{n}\right\}$, we say that a graph property $\mathcal{P} \in \mathbf{L}^{c}$ is $H$-reducible over $\mathbf{L}^{c}$ if there exist non-trivial properties $\mathcal{P}_{1}, \ldots, \mathcal{P}_{n} \in \mathbf{L}^{c}$ such that $H\left[\mathcal{P}_{1}, \ldots, \mathcal{P}_{n}\right]$ is a proper $H$-factorization of $\mathcal{P}$. Otherwise, $\mathcal{P}$ is called $H$-irreducible over $\mathbf{L}^{c}$.

If $H\left[\mathcal{P}_{1}, \ldots, \mathcal{P}_{n}\right]=H\left[\mathcal{P}_{1}^{\prime}, \ldots, \mathcal{P}_{m}^{\prime}\right]=\mathcal{P}$ and there exists an automorphism $\varphi$ of $H$ satisfying $\mathcal{P}_{\varphi(i)}^{\prime}=\mathcal{P}_{i}$ we do not distinguish between these two $H$-factorizations.

Let $\mathcal{C} \subseteq$ PRIME. A graph $H$ on at least two vertices is said to be a $\mathcal{C}$-graph if all base graphs used in the unique construction of $H$ in accordance with Remark 1 are from $\mathcal{C}$. Moreover, we adopt the convention that for each $\mathcal{C} \subseteq$ PRIME, the graph $K_{1}$ is a $\mathcal{C}$-graph. For instance, being a co-graph is equivalent to being a $\left\{K_{2}, \overline{K_{2}}\right\}$-graph.

A graph property $\mathcal{P} \in \mathbf{L}^{c}$ is $\mathcal{C}$-reducible over $\mathbf{L}^{c}$ if $\mathcal{P}$ is $H$-reducible over $\mathbf{L}^{c}$ for some $H$ being a $\mathcal{C}$-graph on at least two vertices, otherwise $\mathcal{P}$ is called $\mathcal{C}$-irreducible over $\mathbf{L}^{c}$. Each $H$-factorization of $\mathcal{P}$ over $\mathbf{L}^{c}$, where $H$ is a $\mathcal{C}$-graph, is said to be a $\mathcal{C}$-factorization of $\mathcal{P}$ over $\mathbf{L}^{c}$. A $\mathcal{C}$-factorization of $\mathcal{P}$ over $\mathbf{L}^{c}$ is irreducible if all its factors are $\mathcal{C}$-irreducible over $\mathbf{L}^{c}$.

The first part of the statement of Lemma 1 allows us to deduce the theorem below.

Theorem 3. If a graph property $\mathcal{P}$ is $\{H\}$-reducible over $\mathbf{L}^{c}$ for some prime graph $H$ then $\mathcal{P}$ is $\left\{H_{1}\right\}$-irreducible over $\mathbf{L}^{c}$ for each prime graph $H_{1}$ which is not isomorphic to $H$.

Proof. If $\mathcal{P}$ is $\{H\}$-reducible and $\left\{H_{1}\right\}$-reducible over $\mathbf{L}^{c}$ for prime graphs $H$ and $H_{1}$, then $H=H_{1}$ by Lemma 1 .

The problems of $\{H\}$-reducibility, over $\mathbf{L}^{c}$ and over other classes of graph properties were earlier discussed only for $H=K_{2}$ and $H=\overline{K_{2}}[7,8,9,10,11,12,13]$. The second one (see [8]) only over the class $\mathbf{L}^{a}$ of additive hereditary graph properties (which is closed under 
disjoint unions of graphs) because in such a class $\left\{\overline{K_{2}}\right\}$-reducibility is equivalent to being a join of incomparable properties from the lattice $\left(\mathbf{L}^{a}, \subseteq\right)[1]$. Properties which are $\left\{K_{2}\right\}$ reducible over a given class of properties always have an irreducible $\left\{K_{2}\right\}$-factorization over those class of graph properties unlike $\{H\}$-reducibility in general. We support this observation by showing examples of hereditary compositive properties which are $\left\{\overline{K_{2}}\right\}$ reducible over $\mathbf{L}^{c}$ but which have no irreducible $\left\{\overline{K_{2}}\right\}$-factorizations over $\mathbf{L}^{c}$. In order to do so, we need some definitions.

Let $\mathcal{C} \subseteq$ PRIME. A graph property $\mathcal{P}_{1}$ is $\mathcal{C}$-infinite over $\mathbf{L}^{c}$ if there exists an infinite sequence of graphs $H_{i}$ in $\mathcal{C}, i \in \mathbb{N}$, and an infinite sequence of hereditary compositive graph properties $\mathcal{P}_{i}, i \in \mathbb{N}$, such that $H_{i}\left[\mathcal{P}_{j_{1}}, \ldots, \mathcal{P}_{j_{n_{i}}}\right]$ with $\mathcal{P}_{j_{n_{i}}}=\mathcal{P}_{i+1}$ is a factorization of $\mathcal{P}_{i}$ over $\mathbf{L}^{c}$ (here $n_{i}$ is the number of vertices of $H_{i}$ ). Otherwise, $\mathcal{P}_{1}$ is $\mathcal{C}$-finite over $\mathbf{L}^{c}$.

For example the properties $\mathcal{S}_{2}=\{G \in \mathcal{I}: \Delta(G) \leq 2\}, \mathcal{P}^{*}=\{G \in \mathcal{I}$ : each component of $G$ is a subgraph of $\left.C_{3}\right\}$ are $\left\{\overline{K_{2}}\right\}$-infinite over $\mathbf{L}^{c}$. It is so because $\mathcal{S}_{2}=$ $\overline{K_{2}}\left[\mathcal{Q}_{3}, \overline{K_{2}}\left[\mathcal{Q}_{4}, \overline{K_{2}}\left[\mathcal{Q}_{5}, \ldots\right]\right]\right]$ and $\mathcal{P}^{*}=\overline{K_{2}}\left[\mathcal{Q}^{*}, \overline{K_{2}}\left[\mathcal{Q}^{*}, \overline{K_{2}}\left[\mathcal{Q}^{*}, \ldots\right]\right]\right]$, where $\mathcal{Q}_{i}=\{G \in \mathcal{I}$ : each component of $G$ is a subgraph of $\left.C_{i}\right\}$ and $\mathcal{Q}^{*}=\left\{G \in \mathcal{I}: G\right.$ is a subgraph of $\left.C_{3}\right\}$.

It is very interesting that $S_{2}=\overline{K_{2}}\left[\mathcal{Q}_{3}, \mathcal{R}\right]$, where $\mathcal{R}=\{G \in \mathcal{I}$ : for each component $G^{\prime}$ of $G$ there is $i \in \mathbb{N} \backslash\{1,2,3\}$ such that $\left.G^{\prime} \subseteq C_{i}\right\}$. This means that $\mathcal{S}_{2}$ has a proper $\overline{K_{2}}$-factorization over $\mathbf{L}^{c}$ and hence it is $\left\{\overline{K_{2}}\right\}$-reducible over $\mathbf{L}^{c}$. On the other hand $\mathcal{P}^{*}$ is $\left\{\overline{K_{2}}\right\}$-irreducible over $\mathbf{L}^{c}$. To observe it, suppose that for some $s \in \mathbb{N}, s \geq 2$, the factorization $\mathcal{P}^{*}=\overline{K_{s}}\left[\mathcal{P}_{1}, \ldots, \mathcal{P}_{s}\right]$ over $\mathbf{L}^{c}$ is proper. Now, for each $i \in \mathbb{N}$ there is a finite number $j_{i}$ such that $j_{i} C_{3} \notin \mathcal{P}_{i}$, otherwise $\mathcal{P}_{i}=\mathcal{P}^{*}$, contrary to the fact that the factorization is proper. It means that $k C_{3} \notin \mathcal{P}^{*}$, for $k=\sum_{i \in[s]} j_{i}$, which is impossible by the definition of $\mathcal{P}^{*}$.

Remark 4. Let $\mathcal{C}_{1} \subseteq \mathcal{C}_{2} \subseteq$ PRIME. If a graph property $\mathcal{P}$ is $\mathcal{C}_{2}$-finite over $\mathbf{L}^{c}$, then it is $\mathcal{C}_{1}$-finite over $\mathbf{L}^{c}$.

Using this fact, we can now conclude that both $\mathcal{S}_{2}$ and $\mathcal{P}^{*}$ are $\mathcal{C}$-infinite over $\mathbf{L}^{c}$ for all $\mathcal{C}$ containing $\overline{K_{2}}$.

In the main part of the paper we study irreducible $\mathcal{C}$-factorizations of $\mathcal{P}$ over $\mathbf{L}^{c}$. To obtain such a factorization we use a procedure in which a given reducible graph property is decomposed until we obtain irreducible factors. It is not obvious that such a procedure always terminates after finitely many steps, but the following fact can immediately be observed.

Remark 5. Let $\mathcal{C} \subseteq$ PRIME. Each $\mathcal{C}$-finite over $\mathbf{L}^{c}$ graph property $\mathcal{P}$ has an irreducible $\mathcal{C}$-factorization over $\mathbf{L}^{c}$.

We now consider the $\mathcal{C}$-infiniteness of a graph property on a set $\mathcal{C}$.

Theorem 4. Let $H_{i}, i \in \mathbb{N}$, be an infinite sequence of graphs in PRIME and let $\mathcal{P}_{i}$, $i \in \mathbb{N}$, be hereditary graph properties such that $H_{i}\left[\mathcal{P}_{i_{1}}=\mathcal{P}_{i+1}, \ldots, \mathcal{P}_{i_{n_{i}}}\right]$ is a factorization of $\mathcal{P}_{i}$ over $\mathbf{L}$. If there exists an infinite subsequence $H_{n_{i}}, i \in \mathbb{N}$, such that $H_{n_{i}} \neq \overline{K_{2}}$ for each $i$, then $\mathcal{P}_{1}$ is the trivial graph property $\mathcal{I}$.

Proof. We shall show that $K_{q} \in \mathcal{P}_{1}$ for each $q \in \mathbb{N}$ which implies that $\mathcal{P}_{1}=\mathcal{I}$. Let $\left\{i_{k}\right\}_{k \in \mathbb{N}}$ be an infinite increasing sequence of positive integers such that $H_{i_{k}} \neq \overline{K_{2}}, k \in \mathbb{N}$. Evidently $K_{1} \in \mathcal{P}_{i}$ for each $i \in \mathbb{N}$, and especially $K_{1} \in \mathcal{P}_{i_{q}}$. Moreover, for each $j>k$ we have $\mathcal{P}_{j} \subseteq \mathcal{P}_{k}$, hence $K_{1} \in \mathcal{P}_{i_{q-1}+1}$. Because $\mathcal{P}_{i_{q-1}}$ has the $H_{i_{q-1}}$-factorization as assumed 
and $\delta\left(H_{i_{q-1}}\right) \geq 1$ ( since $H_{i_{q-1}}$ is different from $\left.\overline{K_{2}}\right)$, it follows that $K_{2} \in \mathcal{P}_{i_{q-1}}$. By the same reasoning $K_{3}$ belongs to $\mathcal{P}_{i_{q-2}}$, etc. Hence $K_{q}$ is an element of $\mathcal{P}_{i_{1}}$ and finally $K_{q} \in \mathcal{P}_{1}$.

Corollary 1. If $\overline{K_{2}} \notin \mathcal{C} \subseteq \mathbf{P R I M E}$ and $\mathcal{P} \in \mathbf{L}^{c}$ is non-trivial, then $\mathcal{P}$ is $\mathcal{C}$-finite over $\mathbf{L}^{c}$.

Using Remark 5, we can now also deduce the following result.

Corollary 2. If $\overline{K_{2}} \notin \mathcal{C} \subseteq \mathbf{P R I M E}$ and $\mathcal{P} \in \mathbf{L}^{c}$ is non-trivial, then $\mathcal{P}$ has an irreducible $\mathcal{C}$-factorization over $\mathbf{L}^{c}$.

We now turn our attention to questions about the irreducibility of $\mathcal{C}$-factorizations for sets $\mathcal{C}$ of prime graphs containing $\overline{K_{2}}$. Before we discuss this, we will prove a lemma which will be useful in later sections too.

Lemma 2. Let $\mathcal{P} \in \mathbf{L}^{c}$ be $\left\{\overline{K_{2}}\right\}$-irreducible over $\mathbf{L}^{c}$ and let $G_{1}$ and $G_{2}$ be two connected graphs which are incomparable in $(\mathcal{I}, \subseteq)$. If $G_{1} \cup G_{2} \in \mathcal{P}$, then there exists a connected graph $G \in \mathcal{P}$ such that $G_{1} \cup G_{2} \subseteq G$.

Moreover, if $\mathcal{P} \in \mathbf{L}^{c}$ is $\left\{\overline{K_{2}}\right\}$-irreducible and $\left\{\overline{K_{2}}\right\}$-finite over $\mathbf{L}^{c}$, then for any two connected graphs $G_{1}$ and $G_{2}$ satisfying $G_{1} \cup G_{2} \in \mathcal{P}$ there is a connected graph $G \in \mathcal{P}$ such that $G_{1} \cup G_{2} \subseteq G$.

Proof. In the first part of the proof we only assume that $G_{1}$ and $G_{2}$ are arbitrary connected graphs in $\mathcal{P}$. Suppose that $\mathcal{P}$ does not contain any connected graph $G$ satisfying $G_{1} \cup G_{2} \subseteq$ $G$. Consider a (finite or infinite) ordered generating set $\mathcal{G}=\left\{H_{1}, H_{2}, H_{3} \ldots\right\}$ for $\mathcal{P}$, such that $G_{1} \cup G_{2} \subseteq H_{1} \subseteq H_{2} \subseteq \ldots$; its existence is guaranteed by Theorem 2. By assumption each graph $H_{i}$ is disconnected and we can assume that it has a form $H_{i}=H_{i}^{1} \cup H_{i}^{2} \cup \cdots \cup$ $H_{i}^{s_{i}}, s_{i} \geq 2, i \in J$, with $H_{i}^{j}$ a connected component of $H_{i}$ for all permissible parameters $i, j$. Then we define a (finite or infinite) graph $T$ with $V(T)=\left\{H_{i}^{j}: i \in J, j \in\left[s_{i}\right]\right\}$ and $E(T)$ constructed in the following way: for each $i \in J$ for which $i+1 \in J$, choose an arbitrary embedding of $H_{i}$ in $H_{i+1}$ (as a subgraph). With respect to that embedding for this fixed $i \in J$ and any $j \in\left[s_{i}\right]$, there is a $k \in\left[s_{i+1}\right]$ such that $H_{i}^{j} \subseteq H_{i+1}^{k}$. For each such set of indices we have an edge $\left\{H_{i}^{j}, H_{i+1}^{k}\right\}$ in $E(T)$. Note that $T$ is a forest. It follows because $H_{i}^{j}$ has to be contained in exactly one component of $H_{i+1}$ for fixed $i, j$. A crucial observation is that $T$ has at least two components. This is a consequence of the fact that $G_{1}$ and $G_{2}$ are contained in different components of $H_{i}$ for each $i \in J$. Moreover, at least two components of $T$ contain vertices which are components of $H_{1}$. Assume that $T$ has components $\left\{T_{q}\right\}_{q \in U}$. Clearly, $U$ can be finite or infinite. Let $W_{i}^{q}=\left\{j \in\left[s_{i}\right]: H_{i}^{j}\right.$ is a vertex of the component $T_{q}$ of $\left.T\right\}$.

We now consider two cases:

(1) $G_{1}, G_{2}$ are incomparable in $(\mathcal{I}, \subseteq)$ and $\mathcal{P}$ is $\left\{\overline{K_{2}}\right\}$-irreducible over $\mathbf{L}^{c}$.

Let $U_{1}$ be the set of indices $q$ in $U$ such that at least one vertex of $V\left(T_{q}\right)$ contains $G_{1}$ as a subgraph. Recall that our construction of $T$ implies that $G_{2}$ is not a subgraph of any vertex in $V\left(T_{q}\right)$, where $q \in U_{1}$. Next, let for all $i, W_{i}^{1}=\cup_{q \in U_{1}} W_{i}^{q}$ and $W_{i}^{2}=\cup_{q \in U \backslash U_{1}} W_{i}^{q}$. Now we construct two properties:

$\mathcal{P}_{1}=\left\{G \in \mathcal{I}: G \subseteq \cup_{j \in W_{i}^{1}} H_{i}^{j}, i \in J\right\}$, and $\mathcal{P}_{2}=\left\{G \in \mathcal{I}: G \subseteq \cup_{j \in W_{i}^{2}} H_{i}^{j}, i \in J\right\}$. The definitions imply that $\mathcal{P}_{1}, \mathcal{P}_{2}$ are hereditary. Further observe that for any $G_{1}^{*}, G_{2}^{*} \in \mathcal{P}_{1}$ one can find indices $i_{1}, i_{2} \in J$, say $i_{i}>i_{2}$, such that $G_{s}^{*} \subseteq \cup_{j \in W_{i_{s}}^{1}} H_{i_{s}}^{j}$, $s \in\{1,2\}$. Thus $\cup_{j \in W_{i_{1}}^{1}} H_{i_{1}}^{j}$ contains both $G_{1}^{*}, G_{2}^{*}$ as subgraphs and hence $\mathcal{P}_{1}$ is 
compositive. Similarly we can prove an analogous fact for $\mathcal{P}_{2}$. By the definition, $\mathcal{P}=\overline{K_{2}}\left[\mathcal{P}_{1}, \mathcal{P}_{2}\right]$ and this $\left\{\overline{K_{2}}\right\}$-factorization of $\mathcal{P}$ over $\mathbf{L}^{c}$ is proper because $G_{1} \cup$ $G_{2} \notin \mathcal{P}_{1} \cup \mathcal{P}_{2}$. This contradicts the $\left\{\overline{K_{2}}\right\}$-irreducibility of $\mathcal{P}$.

(2) To prove the last part of the assertion it is enough to consider the case in which $G_{1} \subseteq G_{2}$ and $\mathcal{P}$ is $\left\{\overline{K_{2}}\right\}$-irreducible and $\left\{\overline{K_{2}}\right\}$-finite over $\mathbf{L}^{c}$.

For each permissible $q$, consider the graph property $\mathcal{P}_{q}=\{G \in \mathcal{I}: G \subseteq$ $\left.\cup_{j \in W_{i}^{q}} H_{i}^{j}, i \in J\right\}$. This defines countable many (finite or infinite) properties, each of which is hereditary and compositive. In the infinite case $\mathcal{P}=\overline{K_{2}}\left[\mathcal{P}_{1}, \overline{K_{2}}\left[\mathcal{P}_{2}, \overline{K_{2}}\left[\mathcal{P}_{3}\right.\right.\right.$, $\left.\left.\left.\overline{K_{2}}[\ldots]\right]\right]\right]$, which means that $\mathcal{P}$ is $\left\{\overline{K_{2}}\right\}$-infinite, a contradiction.

In the finite case we consider the numbers $k, p$ defined in the following way $k=\max \left\{m \in \mathbb{N}: m G_{2} \in \mathcal{P}\right\}$, and $p=|U|$. Evidently $1 \leq k \leq p$. Let $T_{q_{1}}, \ldots, T_{q_{k}}$ be the components of $T$ satisfying that $G_{2}$ is contained in at least one of the vertices of $V\left(T_{q_{i}}\right), i \in[k]$. First we suppose that $k \geq 2$. Hence, assuming that $W_{i}^{*}=\cup_{m \in[k] \backslash\{1\}} W_{i}^{q_{m}}$ we have $\mathcal{P}=\overline{K_{2}}\left[\mathcal{Q}_{1}, \mathcal{Q}_{2}\right]$, where $\mathcal{Q}_{1}=\{G \in$ $\left.\mathcal{I}: G \subseteq \cup_{j \in W_{i}^{*}} H_{i}^{j}, i \in J\right\}, \mathcal{Q}_{2}=\left\{G \in \mathcal{I}: G \subseteq \cup_{j \in\left[s_{i}\right] \backslash W_{i}^{*}} H_{i}^{j}, i \in J\right\}$. The last factorization is proper because $k G_{2} \in \mathcal{P}$ and $k G_{2} \notin \mathcal{Q}_{1} \cup \mathcal{Q}_{2}$. Using the same arguments as before, we can see that the properties $\mathcal{Q}_{1}, \mathcal{Q}_{2}$ are hereditary and compositive, which means that the factorization is over $\mathbf{L}^{c}$. For $k=1$ we construct the proper factorization $\mathcal{P}=\overline{K_{2}}\left[\mathcal{Q}_{1}, \mathcal{Q}_{2}\right]$, where $\mathcal{Q}_{1}=\{G \in \mathcal{I}: G \subseteq$ $\left.\cup_{j \in W_{i}^{q_{1}}} H_{i}^{j}, i \in J\right\}, \mathcal{Q}_{2}=\left\{G \in \mathcal{I}: G \subseteq \cup_{j \in\left[s_{i}\right] \backslash W_{i}^{q_{1}} H_{i}^{j}}, i \in J\right\}$. This factorization, as before, has the desired properties.

Using Lemma 2 we can check at once that $\mathcal{S}_{k}$, for $k \geq 2$, is $\left\{\overline{K_{2}}\right\}$-reducible over $\mathbf{L}^{c}$ because there is no connected graph in $\mathcal{S}_{k}$ containing as subgraphs two graphs which are incomparable in $(\mathcal{I}, \subseteq)$, connected and $k$-regular.

Assume that $\mathcal{S}_{k}, k \geq 2$, has irreducible proper $\left\{\overline{K_{2}}\right\}$-factorization $\overline{K_{s}}\left[\mathcal{Q}_{1}, \ldots, \mathcal{Q}_{s}\right], s \geq 2$, over $\mathbf{L}^{c}$. Using Lemma 2 once again with the $\left\{\overline{K_{2}}\right\}$-irreducibility of $\mathcal{Q}_{i}$ over $\mathbf{L}^{c}$ for each permissible $i$, we can observe that for all $s+1$ graphs $G_{1}, \ldots, G_{s+1}$ which are incomparable in $(\mathcal{I}, \subseteq), k$-regular and connected, we have $G_{1} \cup \cdots \cup G_{s+1} \notin \overline{K_{s}}\left[\mathcal{Q}_{1}, \ldots, \mathcal{Q}_{s}\right]=\mathcal{S}_{k}$, which is in contrast to the definition of $\mathcal{S}_{k}$.

Now we consider the graph property $\mathcal{R}=K_{2}\left[\mathcal{S}_{k}, \mathcal{S}_{k}\right]$. Obviously, the given factorization of $\mathcal{R}$ over $\mathbf{L}^{c}$ is proper because for every two $k$-regular graphs $G_{1}$ and $G_{2}$, the graph $K_{2}\left[G_{1}, G_{2}\right] \in \mathcal{R}$ and $K_{2}\left[G_{1}, G_{2}\right] \notin \mathcal{S}_{k}$. Clearly, by definition, $\mathcal{R}$ is $\left\{K_{2}\right\}$-reducible over $\mathbf{L}^{c}$. Hence, from Theorem 3 it is $\left\{\overline{K_{2}}\right\}$-irreducible over $\mathbf{L}^{c}$. Further, by the same reasoning as before $\mathcal{R}$ is $\left\{K_{2}, P_{5}, \overline{K_{2}}\right\}$-reducible over $\mathbf{L}^{c}$ and still does not possess an irreducible proper $\left\{K_{2}, P_{5}, \overline{K_{2}}\right\}$-factorization over $\mathbf{L}^{c}$. Moreover, it is $\left\{K_{2}, P_{5}, \overline{K_{2}}\right\}$-infinite and $\left\{K_{2}\right\}$-finite over $\mathbf{L}^{c}$. In fact, $\mathcal{R}$ is $\mathcal{C}$-infinite for each $\mathcal{C}$ containing $\left\{\overline{K_{2}}, K_{2}\right\}$.

In general, note that if $\mathcal{P}$ is $\mathcal{C}$-finite over $\mathbf{L}^{c}$ and $\mathcal{P}=H\left[\mathcal{P}_{1}, \ldots, \mathcal{P}_{n}\right]$ is its $\mathcal{C}$-factorization over $\mathbf{L}^{c}$, then each $\mathcal{P}_{i}$ is $\mathcal{C}$-finite over $\mathbf{L}^{c}$. On the other hand, $\mathcal{C}$-infiniteness of each $\mathcal{P}_{i}$ does not imply $\mathcal{C}$-infiniteness of $\mathcal{P}$.

\section{UNIQUENESS}

Lemma 3. If $\mathcal{P} \in \mathbf{L}^{c}$ is $\left\{\overline{K_{2}}\right\}$-irreducible and $\left\{\overline{K_{2}}\right\}$-finite over $\mathbf{L}^{c}$, then the set of all connected graphs in $\mathcal{P}$ is compositive. 
Proof. Suppose that $G_{1}, G_{2}$ are connected graphs in $\mathcal{P}$ having no connected common supergraph in $\mathcal{P}$. Hence, because $\mathcal{P} \in \mathbf{L}^{c}$, we have $G_{1} \cup G_{2} \in \mathcal{P}$. Applying Lemma 2 we obtain a contradiction.

The assertion of the next lemma was stated earlier in [8] for properties, which are $\left\{\overline{K_{2}}\right\}$-irreducible over $\mathbf{L}^{a}$; the proof presented here imitates their proof.

Lemma 4. Let $\mathcal{P} \in \mathbf{L}^{c}$ be $\left\{\overline{K_{2}}\right\}$-irreducible and $\left\{\overline{K_{2}}\right\}$-finite over $\mathbf{L}^{c}$. Then there exists a (finite or infinite) ordered generating set of connected graphs for $\mathcal{P}$.

Proof. Let $\left\{G_{i}: i \in J\right\},(J=[s]$ for some natural $s$ or $J=\mathbb{N})$ be the set of all connected graphs in $\mathcal{P}$. Let $H_{1}=G_{1}$ and, for each $i \in J \backslash\{1\}$, let $H_{i}$ be a connected graph which contains $H_{i-1}$ and $G_{i}$ as subgraphs simultaneously. The existence of such graphs is guaranteed by Lemma 3. Of course $H_{1} \subseteq H_{2} \subseteq \ldots$ Moreover, $\left\{H_{i}: i \in J\right\}$ is a generating set for $\mathcal{P}$ because, in accordance with Lemma 2, each disconnected graph in $\mathcal{P}$ has a connected supergraph and, by the construction, each connected graph in $\mathcal{P}$ is contained in $H_{i}$ for some $i \in J$.

Lemma 5. Let $\mathcal{P}$ be a non-trivial graph property which is $\left\{\overline{K_{2}}\right\}$-finite over $\mathbf{L}^{c}$ and, for $n \geq 2$, let $\overline{K_{n}}\left[\mathcal{P}_{1}, \ldots, \mathcal{P}_{n}\right]$ be an irreducible proper $\left\{\overline{K_{2}}\right\}$-factorization of $\mathcal{P}$ over $\mathbf{L}^{c}$. Then there is no proper $\overline{K_{m}}$-factorization of $\mathcal{P}$ over $\mathbf{L}^{c}$ with $m>n$.

Proof. Suppose, to the contrary, that such a factorization $\overline{K_{m}}\left[\mathcal{P}_{1}^{\prime}, \ldots, \mathcal{P}_{m}^{\prime}\right]$ exists. As we noted earlier properties $\mathcal{P}_{i}$ and $\mathcal{P}_{j}^{\prime}$ are then $\left\{\overline{K_{2}}\right\}$-finite over $\mathbf{L}^{c}$ for all permissible $i, j$. Let $j \in[n]$ be fixed and let $\left\{H_{s}^{j}: s \in L_{j}\right\},\left(L_{j}=\mathbb{N}\right.$ or $L_{j}=\left[k_{j}\right]$ for some natural number $\left.k_{j}\right)$ be an ordered generating set for $\mathcal{P}_{j}$ that consists of only connected graphs. The existence of such sets follows from Lemma 4 . Let $W=\mathbb{N}$ if for at least one $j, L_{j}=\mathbb{N}$ and let $W=\left[\max \left\{k_{j}: j \in[n]\right\}\right]$, otherwise. Put $H_{s}^{j}=H_{\left|L_{j}\right|}^{j}$ for $s \in W, s>\left|L_{j}\right|, j \in[n]$. It is obvious that $\left\{\mathcal{H}_{i}=H_{i}^{1} \cup \cdots \cup H_{i}^{n}: i \in W\right\}$ is a generating set for $\mathcal{P}$. Now, if a graph $G$ has a form $G_{1} \cup G_{2} \cup \cdots \cup G_{n}$ with $G_{i} \in \mathcal{P}_{i}, i \in[n]$, then there exist indices $m_{i}, i \in[n]$, satisfying $G_{i} \subseteq H_{m_{i}}^{i}$ and $m^{*}=\max \left\{m_{i}: i \in[n]\right\}$ and therefore $G \subseteq \mathcal{H}_{m^{*}}$. Since $\overline{K_{m}}\left[\mathcal{P}_{1}^{\prime}, \ldots, \mathcal{P}_{m}^{\prime}\right], m>n$, is a proper factorization of $\mathcal{P}$, there is a $\mathcal{P}$-maximal graph $G^{*}$ satisfying the definition. One can then find an index $q$ such that $G^{*} \subseteq \mathcal{H}_{q}$ which is a supergraph of $G^{*}$ and shows that the corresponding factorization of $\mathcal{P}$ is proper too. This means that $\mathcal{H}_{q}$ has at least $m$ components which gives $n \geq m$, a contradiction.

Now we are in a position to prove the first unique factorization type theorem.

Theorem 5. Each non-trivial graph property $\mathcal{P} \in \mathbf{L}^{c}$ which is $\left\{\overline{K_{2}}\right\}$-finite over $\mathbf{L}^{c}$ has a unique proper irreducible $\left\{\overline{K_{2}}\right\}$-factorization over $\mathbf{L}^{c}$.

Proof. By Lemma 5 it is enough to consider only the proper factorizations of $\mathcal{P}$ with the same number of factors. Let $\overline{K_{n}}\left[\mathcal{P}_{1}, \ldots, \mathcal{P}_{n}\right], \overline{K_{n}}\left[\mathcal{P}_{1}^{\prime}, \ldots, \mathcal{P}_{n}^{\prime}\right]$, be two irreducible proper $\left\{\overline{K_{2}}\right\}$-factorizations of $\mathcal{P}$ over $\mathbf{L}^{c}$. We shall show that there exists a permutation $\sigma$ : $[n] \rightarrow[n]$, such that $\mathcal{P}_{i}^{\prime}=\mathcal{P}_{\sigma(i)}$. Let $\left\{\mathcal{H}_{j}=H_{j}^{1} \cup \cdots \cup H_{j}^{n}: j \in W\right\}$ be a generating set for $\mathcal{P}$, which was constructed as in Lemma 5 using the ordered generating sets $\left\{H_{j}^{i}, j \in W\right\}$ for $\mathcal{P}_{i}, i \in[n]$, of connected graphs. Without loss of generality we can assume by Theorem 2 that each graph $\mathcal{H}_{j}$ is a supergraph of graphs $L_{1}$ and $L_{2}$ simultaneously, where $L_{1}$ and $L_{2}$ are graphs showing that the factorizations $\overline{K_{n}}\left[\mathcal{P}_{1}^{\prime}, \ldots, \mathcal{P}_{m}^{\prime}\right]$ and $\overline{K_{n}}\left[\mathcal{P}_{1}, \ldots, \mathcal{P}_{n}\right]$ are proper factorizations of $\mathcal{P}$ respectively. It implies that in an arbitrary $K_{n}\left[\mathcal{P}_{1}^{\prime}, \ldots \mathcal{P}_{n}^{\prime}\right]$-partition 
of $\mathcal{H}_{j}$ all partition parts are non-empty. It means that for each $j \in W$ there exists a permutation $\sigma_{j}:[n] \rightarrow[n]$ satisfying $H_{j}^{i} \in \mathcal{P}_{\sigma_{j}(i)}^{\prime}$. The set of all permutations of the set $[n]$ is finite. If $W$ is infinite then at least one permutation in the sequence $\left\{\sigma_{j}, j \in W\right\}$ is repeated infinitely many times. In that case let $\sigma$ be such a repeated permutation and let $\sigma=\sigma_{|W|}$ if $W$ is finite. Moreover, let $W^{*}=\left\{j \in W: \sigma=\sigma_{j}\right\}$. Obviously, the set of all $\mathcal{H}_{j}$, satisfying $j \in W^{*}$ is a generating set for $\mathcal{P}$. It is so because for each $G \in \mathcal{P}$ there exists an index $k$ such that $G \subseteq \mathcal{H}_{k}$, and then there exists an index $j>k$ satisfying $\sigma_{j}=\sigma$. By the same reasoning and the construction we know that for an arbitrary $i \in[n]$, the set $\left\{H_{j}^{i}, j \in W^{*}\right\}$ creates the generating set for $\mathcal{P}_{i}$. We shall show that $\mathcal{P}_{s}=\mathcal{P}_{\sigma(s)}^{\prime}$ for each $s \in[n]$. Suppose $G \in \mathcal{P}_{s}$ for fixed $s \in[n]$. Then there exists $k \in W^{*}$ such that $G \subseteq H_{k}^{s}$. But $H_{k}^{s} \in \mathcal{P}_{\sigma(s)}^{\prime}$ so that $\mathcal{P}_{s} \subseteq \mathcal{P}_{\sigma(s)}^{\prime}$. Assume to the contrary that $X \in \mathcal{P}_{\sigma(s)}^{\prime} \backslash \mathcal{P}_{s}$. Moreover let $X^{*}$ be a connected graph in $\mathcal{P}_{s}$ which is not in the graph property $\bar{K}_{n-1}\left[\mathcal{P}_{1}, \ldots, \mathcal{P}_{s-1}, \mathcal{P}_{s+1}, \ldots, \mathcal{P}_{n}\right]$ (its existence follows from the fact that the factorization $\overline{K_{n}}\left[\mathcal{P}_{1}, \ldots, \mathcal{P}_{n}\right]$ is proper, $\left\{\overline{K_{2}}\right\}$-irreducibility of $\mathcal{P}_{s}$ and Lemma 3). Obviously $X^{*} \in \mathcal{P}_{\sigma(s)}^{\prime}$. By the compositivity of $\mathcal{P}_{\sigma(s)}^{\prime}$ there exists a graph $G^{\prime} \in \mathcal{P}_{\sigma(s)}^{\prime}$ which contains both $X$ and $X^{*}$ as subgraphs. Thus by Lemma 4 such a graph is contained in a connected graph $Y$ which is in the graph property $\mathcal{P}_{\sigma(s)}^{\prime}$. Of course $Y \in \mathcal{P}$, consider a $\overline{K_{n}}\left[\mathcal{P}_{1}, \ldots, \mathcal{P}_{n}\right]$-partition of $Y$. It is easy to see that $Y$ has to miss of the properties $\mathcal{P}_{1}, \ldots, \mathcal{P}_{n}$ because of its connectivity. By the construction, and in particular since $X^{*} \subseteq Y$, it must be $\mathcal{P}_{s}$, which contradicts the assumption that $X \notin \mathcal{P}_{s}$.

Given a graph $H$ and two vertices $v_{1}, v_{2}$ of $H$, we say that $v_{1}$ dominates $v_{2}$ in $H$ if $N_{H}\left(v_{2}\right)$ is a proper subset of $N_{H}\left(v_{1}\right), v_{1}$ is a dominating vertex in $H$ if there exists a vertex $v_{2}$ such that $v_{1}$ dominates $v_{2}$ in $H$.

The next lemma plays a key part in the argument for uniqueness in the general case.

Lemma 6. Let $H$ be a prime graph without any dominating vertices with $V(H)=$ $\left\{v_{1}, \ldots, v_{n}\right\}, n \geq 4$. Let $G$ be a fixed graph, $\mathcal{P}_{1}, \ldots, \mathcal{P}_{n} \in \mathbf{L}^{c}$, and let $\left(V_{1}, \ldots, V_{n}\right)$ be an $H\left[\mathcal{P}_{1}, \ldots, \mathcal{P}_{n}\right]$-partition of $G^{*} \in M^{*}\left(H\left[\mathcal{P}_{1}, \ldots, \mathcal{P}_{n}\right]\right)$. Let $X_{k}=G^{*}\left[V_{k}\right]$ if $G \notin \mathcal{P}_{k}$, and let $X_{k}$ be a graph in the graph property $\mathcal{P}_{k}$ and containing both $G^{*}\left[V_{k}\right]$ and $G$ as subgraphs if $G \in \mathcal{P}_{k}$, and let $\hat{G}=H\left[X_{1}, \ldots, X_{n}\right]$. Then each $H\left[\mathcal{P}_{1}, \ldots \mathcal{P}_{n}\right]$-partition of $\hat{G}$ is of the form $\left(V\left(X_{\varphi(1)}\right), \ldots, V\left(X_{\varphi(n)}\right)\right)$ with $\varphi$ an automorphism of $H$ satisfying $G \in \mathcal{P}_{\varphi(i)}$ if and only if $G \in \mathcal{P}_{i}$.

Proof. Let $\left(W_{1}, \ldots, W_{n}\right)$ be an arbitrary $H\left[\mathcal{P}_{1}, \ldots, \mathcal{P}_{n}\right]$-partition of $\hat{G}$. Let $V_{k}^{\prime}$, for $k \in[n]$, be an arbitrary set contained in $V\left(X_{k}\right)$ such that $G^{*}\left[V_{k}\right] \subseteq X_{k}\left[V_{k}^{\prime}\right]$ and $\left|V_{k}\right|=\left|V_{k}^{\prime}\right|$ (actually $G^{*}\left[V_{k}\right]=X_{k}\left[V_{k}^{\prime}\right]$ because $G^{*}\left[V_{k}\right]$ is a $\mathcal{P}_{k}$-maximal graph). According to Lemma 1 there exists an automorphism $\varphi$ of $H$ satisfying $V_{k}^{\prime} \subseteq W_{\varphi(k)}, k \in[n]$. We shall show that $V\left(X_{k}\right)=W_{\varphi(k)}, k \in[n]$. If not, let $v \in V\left(X_{k}\right) \backslash V_{k}^{\prime}$ be a vertex such that $v \in W_{\varphi(l)}$ and $l \neq k$.

(1) There exists $i \in[n] \backslash\{k, l\}$ such that $v_{i}$ is adjacent to $v_{k}$ and it is non-adjacent to $v_{l}$ in $H$. Since $\varphi$ is an automorphism of $H$, we have that $\left\{v_{\varphi(i)}, v_{\varphi(l)}\right\} \notin E(H)$. But, by the construction of $\hat{G}, v$ is adjacent to all vertices of $V_{i}^{\prime}$, where $V_{i}^{\prime} \subseteq W_{\varphi(i)}$, a contradiction.

(2) $N_{H}\left(v_{k}\right) \backslash\left\{v_{l}\right\} \subseteq N_{H}\left(v_{l}\right)$. The facts that $H$ is prime and does not have dominating vertices imply that $\left\{v_{l}, v_{k}\right\} \in E(H)$ and the degree of the vertex $v_{k}$ is smaller 
than the degree of the vertex $v_{l}$. Hence, $G^{*}\left[V_{l}\right]+K_{1} \subseteq \hat{G}\left[V_{l}^{\prime}\right]+K_{1}$. Furthermore, $\hat{G}\left[V_{l}^{\prime}\right]+K_{1}$ is isomorphic to $\hat{G}\left[V_{l}^{\prime} \cup\{v\}\right]$ and this graph is an element of $\mathcal{P}_{l}$ giving that $\left(V_{1}, \ldots, V_{l} \cup\{x\}, \ldots, V_{k} \backslash\{x\}, \ldots, V_{n}\right)$, for an arbitrary $x \in V_{k}$, is an $H\left[\mathcal{P}_{1}, \ldots, \mathcal{P}_{n}\right]$ partition of $G^{*}$, contrary to Lemma 1.

The number of indices $i \in[n]$ such that $W_{i}$ induces in $\hat{G}$ the graph containing $G$ is the same as the number of $i \in[n]$ for which $X_{i}$ has the desired property and it equals the number of indices $i \in[n]$ satisfying $G \in \mathcal{P}_{i}$. This implies the last claim of the theorem.

Theorem 6. Let $H$ be a prime graph on at least three vertices none of which is dominating in $H$. If a graph property $\mathcal{P}$ has a proper $\{H\}$-factorization over $\mathbf{L}^{c}$, then it is unique.

Proof. Let $H\left[\mathcal{P}_{1}, \ldots, \mathcal{P}_{n}\right], H\left[\mathcal{P}_{1}^{\prime}, \ldots, \mathcal{P}_{n}^{\prime}\right]$ be two different proper $H$-factorizations of $\mathcal{P}$ over $\mathbf{L}^{c}$. By Theorem 2, there is a (finite or infinite) ordered generating set $\mathcal{G}=\left\{G_{1}, G_{2} \ldots\right\}$ in which each $G_{i}$ is $\mathcal{P}$-maximal and it is an induced subgraph of $G_{i+1}$. Also, by Theorem 2 , it can be assumed that all graphs $G_{i}$ are from the set $M^{*}\left(H\left[\mathcal{P}_{1}, \ldots, \mathcal{P}_{n}\right]\right) \cap$ $M^{*}\left(H\left[\mathcal{P}_{1}^{\prime}, \ldots, \mathcal{P}_{n}^{\prime}\right]\right)$ with graphs in $\left(\mathcal{G}\left[L_{1}\right]\right)\left[L_{2}\right]$ for $L_{1}, L_{2}$ showing that the given factorizations are proper. Let $\left(V_{i, 1}, \ldots, V_{i, n}\right),\left(\left(V_{i, 1}^{\prime}, \ldots V_{i, n}^{\prime}\right)\right)$ be an arbitrary $H\left[\mathcal{P}_{1}, \ldots \mathcal{P}_{n}\right]$-partition of $G_{i}\left(H\left[\mathcal{P}_{1}^{\prime}, \ldots \mathcal{P}_{n}^{\prime}\right]\right.$-partition $G_{i}$ respectively). By $\varphi_{1}, \ldots, \varphi_{s}$ we denote all automorphisms of $H$. By Lemma 1 there exists an index $l=l\left(G_{i}\right) \in[s]$ satisfying $V_{i, j}^{\prime}=V_{i, \varphi_{l}(j)}, j \in[n]$. We shall now show that there exists an index $p \in[s]$ such that for each permissible $i$ we can find an $H\left[\mathcal{P}_{1}, \ldots, \mathcal{P}_{n}\right]$-partition $\left(W_{i, 1}, \ldots, W_{i, n}\right)$ of $G_{i}$ and an $H\left[\mathcal{P}_{1}^{\prime}, \ldots, \mathcal{P}_{n}^{\prime}\right]$-partition $\left(W_{i, 1}^{\prime}, \ldots, W_{i, n}^{\prime}\right)$ of $G_{i}$ satisfying the equality $W_{i, j}^{\prime}=W_{i, \varphi_{p}(j)}, j \in[n]$.

If $\mathcal{G}$ is the finite $r$-element set then we put $p=r$ and $W_{i, j}=V\left(G_{i}\right) \cap V_{r, j}, j \in[n]$ if $G_{i}$ is isomorphic to $H\left[V\left(G_{i}\right) \cap V_{r, 1}, \ldots, V\left(G_{i}\right) \cap V_{r, n}\right]$ and $W_{i, j}^{\prime}=V\left(G_{i}\right) \cap V_{r, j}^{\prime}, j \in[n]$ if $G_{i}$ is isomorphic to $H\left[V\left(G_{i}\right) \cap V_{r, 1}^{\prime}, \ldots, V\left(G_{i}\right) \cap V_{r, n}^{\prime}\right]$. If $\mathcal{G}$ is infinite then $p$ equals an arbitrary infinitely many times repeated number in the sequence $l\left(G_{1}\right), l\left(G_{2}\right), \ldots$ which is well described because $l\left(G_{i}\right) \in[s]$ for all indices $i$. In this case $W_{i, j}=V\left(G_{i}\right) \cap V_{t, j}, j \in[n]$, where $t$ is an arbitrary index greater then $i$ such that $l\left(G_{t}\right)=p$ and $G_{i}$ is isomorphic to $H\left[V\left(G_{i}\right) \cap V_{t, 1}, \ldots, V\left(G_{i}\right) \cap V_{t, n}\right]$. Similarly $W_{i, j}^{\prime}=V\left(G_{i}\right) \cap V_{t, j}^{\prime}, j \in[n]$, where $t$ is the same index as in the previous line and $G_{i}$ is isomorphic to $H\left[V\left(G_{i}\right) \cap V_{t, 1}^{\prime}, \ldots, V\left(G_{i}\right) \cap V_{t, n}^{\prime}\right]$. In both cases depending on the finiteness of $\mathcal{G}$ the equality $W_{i, j}^{\prime}=W_{i, \varphi_{p}(j)}$ holds, for $j \in[n]$ and all permissible indices $i$.

We continue to show that $\mathcal{P}_{j}^{\prime}=\mathcal{P}_{\varphi_{p}(j)}$ for all $j \in[n]$. Let for the fixed $j \in[n], \mathcal{Q}_{j}$ be a graph property for which $\left\{G_{i}\left[W_{i, j}^{\prime}\right]\right.$ : all permissible $\left.i\right\}$ is a generating set. Of course $\mathcal{Q}_{j} \subseteq \mathcal{P}_{\varphi_{p}(j)}$ and $\mathcal{Q}_{j} \subseteq \mathcal{P}_{j}^{\prime}$. Let us take a look at the inverse inclusions. First suppose that there is an $L \in \mathcal{P}_{j}^{\prime} \backslash \mathcal{Q}_{j}$ for selected $j \in[n]$. Let $q$ be fixed and $\left(W_{q, 1}^{\prime}, \ldots, W_{q, n}^{\prime}\right)$ be a partition as in the paragraph above. Similarly to Lemma 6 , let $Y_{k}=G_{q}\left[W_{q, k}^{\prime}\right]$ if $L \notin \mathcal{P}_{k}^{\prime}$ and $Y_{k}$ be a graph in the graph property $\mathcal{P}_{k}^{\prime}$ which contains both $G_{q}\left[W_{q, k}^{\prime}\right]$ and $L$ as subgraphs if $L \in \mathcal{P}_{k}^{\prime}$. Put $\hat{G}=H\left[Y_{1}, \ldots, Y_{n}\right]$. It then follows by Lemma 6 that an arbitrary $H\left[\mathcal{P}_{1}, \ldots \mathcal{P}_{n}\right]$-partition of $\hat{G}$ is of the form $\left(V\left(Y_{\varphi(1)}\right), \ldots, V\left(Y_{\varphi(n)}\right)\right)$ with $\varphi$ being an automorphism of $H$ satisfying $L \in \mathcal{P}_{\varphi(j)}^{\prime}$ if and only if $L \in \mathcal{P}_{j}^{\prime}$. The next observation is that, because $\mathcal{G}$ is a generating set for $\mathcal{P}$ and $\hat{G} \in \mathcal{P}$, there exists an index $r$ such that $\hat{G} \subseteq G_{r}$. For each $H\left[\mathcal{P}_{1}^{\prime}, \ldots, \mathcal{P}_{n}^{\prime}\right]$-partition of $G_{r}$ we can construct the $H\left[\mathcal{P}_{1}^{\prime}, \ldots, \mathcal{P}_{n}^{\prime}\right]$ partition of $\hat{G}$ taking as a partition part the intersection of the previous one with $V(\hat{G})$. Thus, by Lemma 6 , in each $H\left[\mathcal{P}_{1}, \ldots, \mathcal{P}_{n}\right]$-partition of $G_{r}$, especially in $\left(W_{r, 1}^{\prime}, \ldots, W_{r, n}^{\prime}\right)$, 
we obtain $L \subseteq G_{r}\left[Y_{r, j}\right]$, which proves that $L \in \mathcal{Q}_{j}$, contrary to the assumption. Thus there is no such $L$ and $\mathcal{P}_{j}^{\prime} \subseteq \mathcal{Q}_{j}$. The case where $\mathcal{P}_{\varphi_{p}(j)} \subseteq \mathcal{Q}_{j}$ is dealt with similarly. These arguments yield $\mathcal{P}_{j}^{\prime}=\mathcal{P}_{\varphi_{p}(j)}$ for all $j \in[n]$.

We now show with an example that the assumption about the non-existence of dominating vertices in $H$ is necessary. Consider the prime graph $H=P_{4}$, the path on four vertices, which has dominating vertices. Let $V\left(P_{4}\right)=\left\{v_{1}, v_{2}, v_{3}, v_{4}\right\}$ and $E\left(P_{4}\right)=\left\{\left\{v_{i}, v_{i+1}\right\}: i \in\right.$ [3] $\}$ and let $\mathcal{P}_{1}=\left\{G \in \mathcal{I}\right.$ : each component of $G$ is a subgraph of a cycle $C_{3}$ or $\left.C_{5}\right\}$, $\mathcal{P}_{2}=\mathcal{P}_{4}=\left\{K_{1}\right\}, \mathcal{P}_{3}=\left\{G \in \mathcal{I}\right.$ : each component of $G$ is a subgraph of a cycle $C_{5}$ or $\left.C_{4}\right\}$.

Consider a factorization $P_{4}\left[\mathcal{P}_{1}, \mathcal{P}_{2}, \mathcal{P}_{3}, \mathcal{P}_{4}\right]$ of $\mathcal{P}$. It is easy to see that the graph $P_{4}\left[C_{3}, K_{2}, C_{4}, K_{1}\right]$ confirms that the factorization is proper. However, $\mathcal{P}$ does not have a unique proper factorization since another representation could be $\mathcal{P}=P_{4}\left[\mathcal{P}_{1}^{*}, \mathcal{P}_{2}, \mathcal{P}_{3}, \mathcal{P}_{4}\right]$, where $\mathcal{P}_{1}^{*}=\left\{G \in \mathcal{I}\right.$ : each component of $G$ is a subgraph of $\left.C_{3}\right\}$.

The next theorem complements Theorems 5 and 6 .

Theorem 7. $[9,10]$ Each graph property $\mathcal{P} \in \mathbf{L}^{c}$ has a unique irreducible proper $\left\{K_{2}\right\}$ factorization over $\mathbf{L}^{c}$.

\section{MAin RESUlts}

We now give the two main theorems of the paper and give one short proof for both of them obtained by applying our earlier results.

Theorem 8. Let $\mathcal{C}$ be a family of prime graphs without dominating vertices and let $\overline{K_{2}} \notin \mathcal{C}$. Each non-trivial graph property $\mathcal{P} \in \mathbf{L}^{c}$ has a unique proper irreducible $\mathcal{C}$-factorization over $\mathbf{L}^{c}$.

Theorem 9. Let $\mathcal{C}$ be a family of prime graphs without dominating vertices. If a nontrivial graph property $\mathcal{P} \in \mathbf{L}^{c}$ is $\mathcal{C}$-finite over $\mathbf{L}^{c}$, it has a unique proper irreducible $\mathcal{C}$ factorization over $\mathbf{L}^{c}$.

Proof. By Corollaries 1, 2 and Remark 5 there exists at least one irreducible proper $\mathcal{C}$ factorization of $\mathcal{P}$ over $\mathbf{L}^{c}$.

Assume that $H_{1}\left[\mathcal{P}_{1}^{1}, \ldots, \mathcal{P}_{1}^{n_{1}}\right], H_{2}\left[\mathcal{P}_{2}^{1}, \ldots, \mathcal{P}_{2}^{n_{2}}\right]$ are two different irreducible proper $\mathcal{C}$ factorizations of $\mathcal{P}$ over $\mathbf{L}^{c}$. If at least one of the graphs $H_{1}$ and $H_{2}$ is isomorphic to $K_{1}$, then $\mathcal{P}$ is $\mathcal{C}$-irreducible over $\mathbf{L}^{c}$ and $\mathcal{P}=\mathcal{P}_{1}=\mathcal{P}_{1}^{\prime}$. Next, suppose that $\left|V\left(H_{1}\right)\right| \geq$ $\mid V\left(H_{2} \mid \geq 2\right.$. Remark 1 implies that $H_{i}$ is either disconnected with components $H_{i}^{1}$, $H_{i}^{2}, \ldots, H_{i}^{s_{i}}$ (this case is possible only in the proof of Theorem 9) or $\overline{H_{i}}$ is disconnected with components $\overline{H_{i}^{1}}, \overline{H_{i}^{2}}, \cdots, \overline{H_{i}^{s_{i}}}$ or $H_{i}=G_{i}\left[H_{i}^{1}, \ldots, H_{i}^{s_{i}}\right]$ with $G_{i} \in \mathcal{C} \backslash\left\{K_{2}, \overline{K_{2}}\right\}$, $i \in\{1,2\}$. Of course $H_{i}^{j}$ is a $\mathcal{C}$-graph for $j \in\left[s_{i}\right]$ and $i \in\{1,2\}$. In each case we relable the vertices of $H_{i}$ such that $V\left(H_{i}\right)=\left\{v_{i}^{1}, \ldots, v_{i}^{n_{i}}\right\}$ and $V\left(H_{i}^{1}\right)=\left\{v_{i}^{1}, \ldots, v_{i}^{\left|V\left(H_{i}^{1}\right)\right|}\right\}$, $V\left(H_{i}^{j}\right)=\left\{v_{\sum_{t=1}^{j-1}\left|V\left(H_{i}^{t}\right)\right|+1}, \ldots, v_{\sum_{t=1}^{j}\left|V\left(H_{i}^{t}\right)\right|}\right\}, 2 \leq j \leq s_{i}$. Evidently $n_{i}=\sum_{t=1}^{s_{i}}\left|V\left(H_{i}^{t}\right)\right|$. Moreover, the above forces three mutually exclusive possible forms of a proper factorization of $H_{i}\left[\mathcal{P}_{i}^{1}, \ldots, \mathcal{P}_{i}^{n_{i}}\right]$ (of which only the last two need to be considered for the proof of Theorem 8):

(1) $H_{i}\left[\mathcal{P}_{i}^{1}, \ldots, \mathcal{P}_{i}^{n_{i}}\right]=\overline{K_{s_{1}}}\left[H_{i}^{1}\left[\mathcal{P}_{i}^{1}, \ldots, \mathcal{P}_{i}^{\left|V\left(H_{i}^{1}\right)\right|}\right], \ldots, H_{i}^{s_{i}}\left[\mathcal{P}_{i}^{\sum_{t=1}^{s_{i}-1}\left|V\left(H_{i}^{t}\right)\right|+1}, \ldots, \mathcal{P}_{i}^{n_{i}}\right]\right]$,

(2) $H_{i}\left[\mathcal{P}_{i}^{1}, \ldots, \mathcal{P}_{i}^{n_{i}}\right]=K_{s_{1}}\left[H_{i}^{1}\left[\mathcal{P}_{i}^{1}, \ldots, \mathcal{P}_{i}^{\left|V\left(H_{i}^{1}\right)\right|}\right], \ldots, H_{i}^{s_{i}}\left[\mathcal{P}_{i}^{\sum_{t=1}^{s_{i}-1}\left|V\left(H_{i}^{t}\right)\right|+1}, \ldots, \mathcal{P}_{i}^{n_{i}}\right]\right]$,

(3) $H_{i}\left[\mathcal{P}_{i}^{1}, \ldots, \mathcal{P}_{i}^{n_{i}}\right]=G_{i}\left[H_{i}^{1}\left[\mathcal{P}_{i}^{1}, \ldots, \mathcal{P}_{i}^{\left|V\left(H_{i}^{1}\right)\right|}\right], \ldots, H_{i}^{s_{i}}\left[\mathcal{P}_{i}^{\sum_{t=1}^{s_{i}-1}\left|V\left(H_{i}^{t}\right)\right|+1}, \ldots, \mathcal{P}_{i}^{n_{i}}\right]\right]$. 
Thus, by Theorem 3 , for both the $H_{1}$-factorization and the $H_{2}$-factorization we have the same possibility (either (1), or (2) or (3)). Let $\mathcal{Q}_{i}^{j}=H_{i}^{j}\left[\mathcal{P}_{i}^{\sum_{t=1}^{j-1}\left|V\left(H_{i}^{t}\right)\right|+1}, \ldots, \mathcal{P}_{i}^{\sum_{t=1}^{j}\left|V\left(H_{i}^{t}\right)\right|}\right]$ for all permissible $i, j$. In case $(1)((2))$, Remark 1 , the assumption about $\left\{\overline{K_{2}}\right\}$-irreducibility ( $\left\{K_{2}\right\}$-irreducibility) of $\mathcal{P}_{i}^{j}$ (applied for $H_{i}^{j}=K_{1}$ ) and the above consideration lead to the assertion that $\mathcal{Q}_{i}^{j}$ is $\left\{\overline{K_{2}}\right\}$-irreducible $\left(\left\{K_{2}\right\}\right.$-irreducible respectively) for all permissible $i, j$. Moreover, in case (1), Remark 4 implies that each $\mathcal{Q}_{i}^{j}$ as a graph property which is $\mathcal{C}$-finite over $\mathbf{L}^{c}$, is $\left\{\overline{K_{2}}\right\}$-finite over $\mathbf{L}^{c}$. Hence, in accordance with Theorem 5 (Theorem 7), $s_{1}=s_{2}=s$ and there exists a permutation $\varphi$ satisfying $\mathcal{Q}_{2}^{j}=\mathcal{Q}_{1}^{\varphi(j)}$ for all $j \in[s]$. In case (3), by Theorem 3, $G_{1}$ is isomorphic to $G_{2}$ and by Theorem 6 there exists an automorphism $\varphi$ of $G_{1}$ such that $\mathcal{Q}_{2}^{j}=\mathcal{Q}_{1}^{\varphi(j)}$ for all $j \in[s]$. We apply the same reasoning for all $\mathcal{Q}_{i}^{j}$ which are $\mathcal{C}$-reducible over $\mathbf{L}^{c}$. This procedure terminates by the assumption that $\mathcal{P}$ is $\mathcal{C}$-finite over $\mathbf{L}^{c}$. Finally we compose all automorphisms and permutations obtained from this procedure in order to observe that $H_{1}$ is isomorphic to $H_{2}$. At the same time this shows that there exists an automorphism $\varphi$ of $H_{1}$ such that $\mathcal{P}_{2}^{j}=\mathcal{P}_{1}^{\varphi(j)}$.

\section{REFERENCES}

[1] Borowiecki, M., Mihók, P.: Hereditary properties of graphs. in: Kulli, V.R. ed., Advance in Graph Theory (Vishawa International Publication), Gulbarga, 41-68, 1991

[2] Cockayne, E.J.: Colour classes for r-graphs. Canad. Math. Bull. 15, 349-354(1972)

[3] Hell, P. and Nešetřil, J., Graphs and Homomorphisms, Oxford Lecture Series in Mathematics and Its Applications, Oxford University Press, 2004

[4] Jakubík, J.: On the Lattice of Additive Hereditary Properties of Finite Graphs. Discussiones Mathematicae General Algebra and Applications 22, 73-86(2002)

[5] Drgas-Burchardt, E.: On uniqueness of a general factorization of graph properties. Journal of Graph Theory 62, 48-64(2009)

[6] Drgas-Burchardt, E.: Cardinality of a minimal forbidden graph family for reducible additive hereditary graph properties. Discussiones Mathematicae Graph Theory 29(2), 263-274(2009)

[7] Berger, A.J.: Minimal forbidden subgraphs of reducible graph properties. Discussiones Mathematicae Graph Theory 21, 111-117(2001)

[8] Berger, A.J., Broere, I., Moagi, S.J.T., Mihók, P.: Meet- and join-irreducibility of additive hereditary properties of graphs. Discrete Mathematics 251, 11-18(2002)

[9] Farrugia, A.: Uniqueness and complexity in generalised coloring, $\mathrm{PhD}$ thesis, Waterloo, Ontario, Canada, 2003

[10] Farrugia, A., Mihók, P., Richter, R. Bruce, Semanišin, G.: Factorizations and characterizations of induced-hereditary and compositive properties. Journal of Graph Theory 49, 11-27(2000)

[11] Farrugia, A., Richter, R. Bruce: Unique factorisation of additive induced-hereditary properties. Discussiones Mathematicae Graph Theory 24, 319-343(2004)

[12] Mihók, P., Semanišin, G., Vasky, R.: Additive and hereditary properties of graphs are uniquely factorizable into irreducible factors. Journal of Graph Theory 33, 44-53(2000)

[13] Zverovich, I.E.: $r$-Bounded $k$-complete bipartite bihypergraphs and generalized split graphs. Discrete Mathematics 247, 261-270(2002)

[14] Gallai, T.: Transitiv orientierbare Graphen. Acta Mathematica Academiae Scientiarum Hungaricae 18, 25-66(1967)

[15] James, L.O., Stanton, R.G., Cowan, D.D.: Graph decomposition for undirected graphs. in: Proceedings of the Third Southeastern International Conference on Combinatorics, Graph Theory and Computing (CGTC'72), 281-290, 1972 